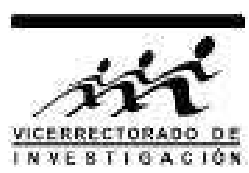

\title{
Análisis mineralógico de la fracción arcilla de suelos tropicales del Perú por difractometría de rayos $X$ y espectroscopia Mössbauer
}

\author{
Mirian E. Mejia* y Jorge A. Bravo \\ Facultad de Ciencias Físicas, Universidad Nacional Mayor de San Marcos, A.P. 14-0149, Lima 14, Perú
}

Recibido 08 mayo 2014 - Aceptado 10 junio 2014

\begin{abstract}
El propósito de este trabajo fue la identificación y la caracterización de los principales minerales en las fracciones arcilla de muestras de suelos tropicales que fueron seleccionadas de la Reserva Forestal de la Universidad Agraria de la Selva, localizada en la Región de Huánuco, Perú, con énfasis en el estudio de los sesquióxidos de hierro (óxidos, hidróxidos y oxidróxidos de hierro) y minerales de arcilla con sustitución de hierro. Se utilizó el método químico de disolución selectiva utilizando ditionito-citrato-bicarbonato, DCB, [1] para la disolución de óxidos de hierro cristalinos, lo cual favorece la identificación de minerales de arcilla. Asimismo fueron utilizadas las técnicas analíticas de difractometría de rayos X, DRX, y espectroscopia Mössbauer por transmisión, EMT, a temperatura ambiente, TA, y a $110 \mathrm{~K}$ para determinar la composición mineralógica de las muestras. En este artículo presentamos solo los resultados obtenidos con dos muestras seleccionadas por sus características contrastantes.
\end{abstract}

Palabras claves: fraccion arcilla, sesquióxidos de hierro, difractometria de rayos $\mathrm{X}$, espectroscopia Mössbauer.

\section{Mineralogical analysis of the clay fraction of tropical soils from Peru by X-ray diffracto- metry and Mössbauer spectroscopy}

The purpose of this work was to identify and to some extent characterize the main minerals in the clay fractions of soil samples from the Forest Reserve of the Universidad Agraria de la Selva, located in the Region of Huánuco, Peru, with emphasis on the iron oxides and iron-bearing clay minerals, using chemical dissolutive methods, such as dithionite-citrate-bicarbonate, DCB, [1] to dissolve preferentially crystalline iron oxides, and analytical techniques such as $\mathrm{X}$ ray diffractometry, XRD, and transmission Mössbauer spectroscopy, TMS, at room temperature, RT, and at $110 \mathrm{~K}$ was used to determine their mineralogical composition. This paper details the results obtained with two selected samples with contrasting characteristics.

Keywords: clay fraction, iron oxides, X-ray diffractometry, Mössbauer spectroscopy.

En la Región Amazónica Peruana sólo el $2.9 \%$ de los suelos son actualmente usados como tierras de cultivo y el $7.6 \%$ son usados como pastizales; y alrededor del $89.5 \%$ es potencialmente para explotación forestal. Las muestras estudiadas en este trabajo fueron colectadas del área nativa de la Reserva Forestal de la Universidad Nacional de la Selva, BRUNAS, con coordenadas geográficas de $9^{\circ} 09^{\prime} \mathrm{S}$ y $75^{\circ} 35^{\prime} \mathrm{O}$, localizada en el área tropical de Tingo María, Región de Huánuco, Perú, ver Figura 1] a una altitud de 1100 msnm, dentro de un área montañosa con lluvias anuales de $3079 \mathrm{~mm}$ y una temperatura promedio de $23^{\circ} \mathrm{C}$. De las muestras estudiadas se seleccionaron las correspondientes a Supte y Tornillo debido a sus características contrastantes, por el diferente porcentaje en contenido hierro y por la presencia de hierro en estado superparamagnético. Las muestras de suelo fueron colectadas a una profundidad de $0.30 \mathrm{~m}$ desde la superficie del suelo. El análisis mineralógico de las fracciones arcillosas, que fueron separadas por sedimentación de acuerdo al procedimiento descrito por Jackson [1], se llevó a cabo usando difractometría de rayos X, DRX [2,3] y espectroscopia Mössbauer por transmisión, EMT [4].

Debido a la superposición de algunos picos de reflexión de rayos $X$ del cuarzo y de minerales de arcilla, así como también a la baja concentración de los minerales de arcilla en la fracción arcillosa, es difícil distinguir las reflexiones de los minerales de arcilla, aún más si éstos están recubiertos de sesquióxidos de hierro [5] y aluminio. La aplicación de la técnica de DCB permite que las reflexiones de los minerales de arcilla se intensifiquen. Por EMT es posible

*mmejiam@gmail.com 
obtener más información acerca de los minerales que contienen hierro por sustitución catiónica, indiferentemente de su estado amorfo o cristalino, sin interferencia de otros minerales que no contienen hierro.

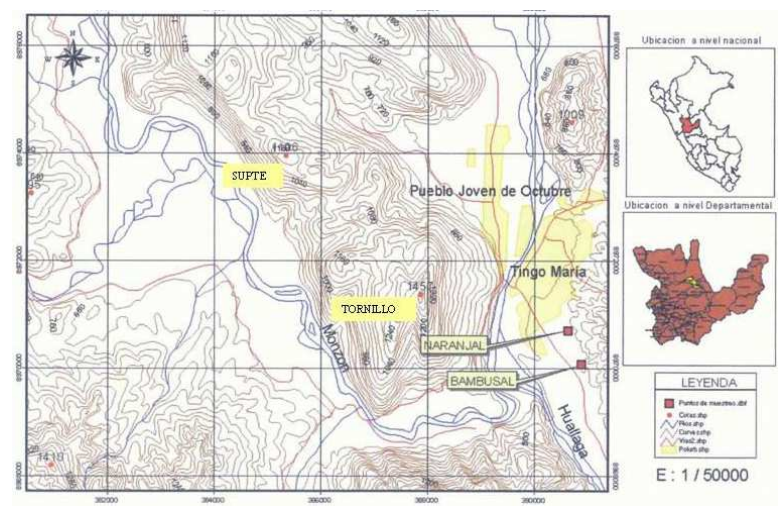

Figura 1: Mapa geológico de la región de Tingo María. Los círculos rojos muestran el área en estudio.

\section{Métodos Experimentales}

Las dos muestras de suelos seleccionadas fueron etiquetadas como Supte y Tornillo. Las fracciones arcillosas menores a $2 \mu \mathrm{m}$ de estas muestras fueron obtenidas por el proceso de sedimentación o método del hidrómetro. Para tal proceso se utilizó $50 \mathrm{~g}$ de muestra de suelo a la cual se agregó unos $5 \mathrm{ml}$ de un agente dispersante, hexametafosfato de sodio y carbonato de sodio, dejando embeber la muestra por una noche; luego se agregó unos $700 \mathrm{ml}$ de agua destilada; la solución agua-suelo obtenida se agitó por unos 15 min para después ser vertida en un bouyouco, el cual se enrasó al nivel superior de $1250 \mathrm{ml}$ con agua destilada; después de $7 \mathrm{~h}$ de reposo se sifoneó el sobrenadante para ser llevado a secar en una mufla a $60^{\circ} \mathrm{C}$.

Las fracciones arcillosas obtenidas fueron sometidas a un tratamiento químico de disolución selectiva de sesquióxidos de Fe cristalinos usando ditionito- citratobicarbonato según el método de Mehra Jackson [6]. Después de este tratamiento las muestras fueron lavadas para eliminar las sales y secadas a una temperatura no superior de $45^{\circ} \mathrm{C}$, obteniéndose un total de cuatro muestras, las fracciones arcillosas de Supte y Tornillo con y sin tratamiento DCB.

Las muestras en polvo fueron analizadas por DRX. La radiación utilizada fue de rayos- $X$ provenientes de Co$\mathrm{K} \alpha$, aplicando $32.5 \mathrm{kV}$ y $25 \mathrm{~mA}$ en los rangos de $4^{\circ}<2 \theta$ $<80^{\circ}$ a barrido continuo. Además, se utilizó un espectrómetro convencional para el analisis por EMT, consistente de una fuente de $50 \mathrm{mCi}$ de ${ }^{57} \mathrm{Co}$ en matriz de Rh. Los espectros fueron tomados a temperatura ambiente (TA) y a $110 \mathrm{~K}$; se utilizó $250 \mathrm{mg}$ y $100 \mathrm{mg}$ de las muestras para las medidas a TA y $110 \mathrm{~K}$ respectivamente. El ajuste de los espectros se realizó con la ayuda del programa de ajuste Normos [7]. Los parámetros hiperfinos obtenidos se muestran en la Tabla 1

\section{Medidas de $\mathrm{pH}$ y determinación del valor Munsell}

Los resultados de las medidas de $\mathrm{pH}$ de la muestra Supte corresponde a un suelo ligeramente ácido, $\mathrm{pH}=6.4$, mientras que la muestra Tornillo corresponde a un suelo relativamente ácido, $\mathrm{pH}=4.6$. Con la ayuda de la cartilla Munsell, edición 2009, se determinó el color de las muestras; para el caso de la muestra Supte le corresponde el código Munsell 10 YR (7/4) asignado al color marrón muy pálido, y para la muestra Tornillo le corresponde el código Munsell 7,5 YR (6/3) asignado al color marrón claro.

\section{Análisis por difractometría de rayos $\mathrm{X}$}

En la Figura 2 presentamos los difractogramas de DRX de las fracciones arcillosas de Supte con y sin tratamiento DCB. La fracción sin DCB muestra la ocurrencia de fases no magnéticas tales como cuarzo y caolinita, y sesquióxidos de Fe ordenados magnéticamente como hematita y goethita. En la fracción tratada con DCB se aprecia una disminución en la intensidad de las reflexiones de la hematita, así como la ocurrencia de reflexiones características débiles de fases mineralógicas de clorita y partheita, $\mathrm{Ca}_{2} \mathrm{Al}_{4} \mathrm{Si}_{4} \mathrm{O}_{15}(\mathrm{OH})_{2}\left(\mathrm{H}_{2} \mathrm{O}\right)_{4}$, lo cual indica que gracias al tratamiento DCB fue posible observar reflexiones características débiles que no fue posible observar en la muestra no tratada.

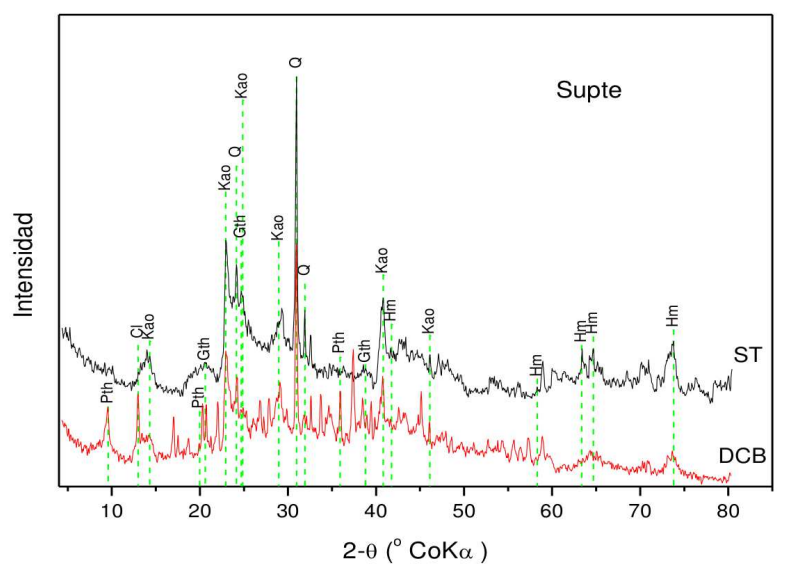

Figura 2: DRX de la fracción arcillosa Supte sin tratar y tratada con DCB. Los acrónimos son $\mathrm{P} t h=$ partheite, $\mathrm{Cl}=$ clorita, $\mathrm{Kao}=$ caolinita, $\mathrm{Q}=$ cuarzo, $\mathrm{Hm}=$ hematita, $\mathrm{Gth}=$ goethita, $\mathrm{V}=$ vermiculita, Mont $=$ montmorillonita, Mus $=$ moscovita .

En la Figura 3 se muestran los difractogramas de la fracción arcillosa de la muestra Tornillo con y sin tratamiento DCB. A diferencia de la fracción arcillosa Supte, esta muestra presenta menor concentración de la fase de 
cuarzo. En la fracción no tratada con DCB, se observa la ocurrencia de alúmino silicatos como illita y moscovita, y minerales interestratificados de caolinita-montmorillonita y clorita-vermiculita-montmorillonta que siguen aún presentes después que la muestra fue tratada con DCB. Las reflexiones de los sesquióxidos de Fe sufren disminución en sus intensidades después del tratamiento con DCB. La presencia de la fase partheita está también presente en la fracción tratada como se observa en la muestra Supte. Cabe mencionar que no se pudo asignar las reflexiones características observadas a $12.88^{\circ}$ y $20.26^{\circ}(2 \theta)$ a ningún mineral comúnmente encontrado en un suelo.

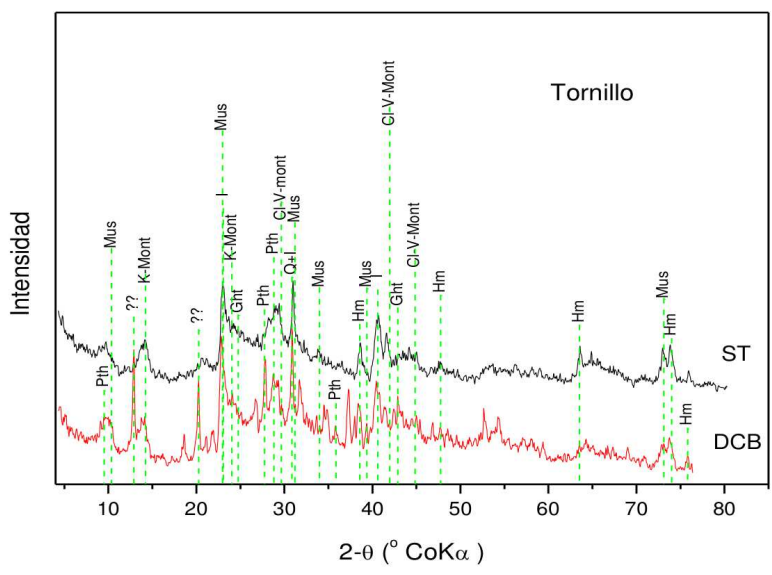

Figura 3: DRX de la fracción arcillosa de la muestra de Tornillo sin tratar y tratada con DCB. Los acrónimos son $\mathrm{Pth}=$ partheite, $\mathrm{Cl}=$ clorita, $\mathrm{K}=$ caolinita, $\mathrm{Q}=$ cuarzo, $\mathrm{Hm}=$ hematita, $\mathrm{Gth}=$ goethita, $\mathrm{V}=$ vermiculita, Mont $=$ montmorillonita, Mus = moscovita.

\section{Espectros Mössbauer}

Los espectros Mössbauer de las fracciones arcilla con y sin tratamiento DCB, fueron tomados a temperatura ambiente y a la temperatura $\mathrm{T}=110 \mathrm{~K}$. Los correspondientes espectros las muestras de Subte y Tornillo se muestran en la Figuras 4.

Los espectros de las muestras Supte no tratada y tratada a TA fueron ajustados con una incipiente componente magnética y dos dobletes asignados a sitios de $\mathrm{Fe}^{3+}$; los dobletes de $\mathrm{Fe}^{3+}$ pueden ser debidos al hierro estructural de los minerales de arcilla y/o a los sesquióxidos de hierro en estado superparamagnético. Como resultado del tratamiento químico, el área total de resonancia decrece alrededor del $62 \%$; la presencia del pequeño doblete a TA está relacionado a la goethita en estado superparamagnético. El espectro a $\mathrm{T}=110 \mathrm{~K}$ de la muestra tratada con DCB fue ajustada con un sexteto asignado a la hematita, una distribución de campo magnético asignada a la goethita, un doblete paramagnético asignado al sitio de $\mathrm{Fe}^{3+}$ y dos dobletes paramagnéticos asignados a sitios de $\mathrm{Fe}^{2+}$. Para el caso de la muestra no tratada el ajuste por distribución contiene un área total de aproximadamente $72 \%$ que es asignada a la goethita. El tratamiento con DCB causa que el área total de resonancia decrezca alrededor del $47 \%$ y afecta la distribución relativa de las áreas de los sitios, mientras que el área relativa de la goethita disminuye proporcionalmente, en concordancia con la interpretación dada a los espectros tomados a TA. Los valores correspondientes a los parámetros hiperfinos se muestran para las muestras Supte y Tornillo en la Tabla 1.

En el caso de la muestra Tornillo, los espectros tomados a TA para la muestra no tratada y tratada, fueron ajustados con dos sitios magnéticos y dos dobletes paramagnéticos; uno de los sitios magnéticos está caracterizado por un ancho de línea estrecho y un campo hiperfino mayor; mientras que el segundo sitio magnético está caracterizado por un ancho de línea grande y un campo hiperfino menor, el cual está probablemente asociado a una fase amorfa; ambos sextetos son asignados a la hematita; uno de los dobletes fue asignado a un sitio de $\mathrm{Fe}^{3+}$ y el otro, el cual es mucho más débil, se le asignó a un sitio de $\mathrm{Fe}^{2+}$. Para la muestra no tratada el área de los sextetos da cuenta de alrededor del $53 \%$ del área total. El tratamiento químico causa que el área total decrezca alrededor del $8 \%$, debido a la remoción del hierro de todos los sitios; observándose en el espectro un incremento de la intensidad relativa del sexteto de mayor campo hiperfino. Para el ajuste de los espectros tomados a $110 \mathrm{~K}$, fue necesario incluir un sitio magnético, dos dobletes y una distribución de campo hiperfino. Uno de los dobletes fue asignado al sitio de $\mathrm{Fe}^{3+}$ y el otro fue asignado al sitio de $\mathrm{Fe}^{2+}$. Los sitios de estos dobletes pueden estar localizados en los minerales arcillosos; el doblete más intenso podría estar asociado a moscovita, illita o minerales interestratificados, como se evidencia por DRX. Debido al tratamiento con DCB se puede apreciar un crecimiento del área magnetica por sitio en alrededor de un $30 \%$, y una disminución del área magnética asociada a la distribución, la cual está asociada a la goethita. Los valores correspondientes de los parámetros hiperfinos de la muestra Tornillo se muestran en la Tabla 1 ] 

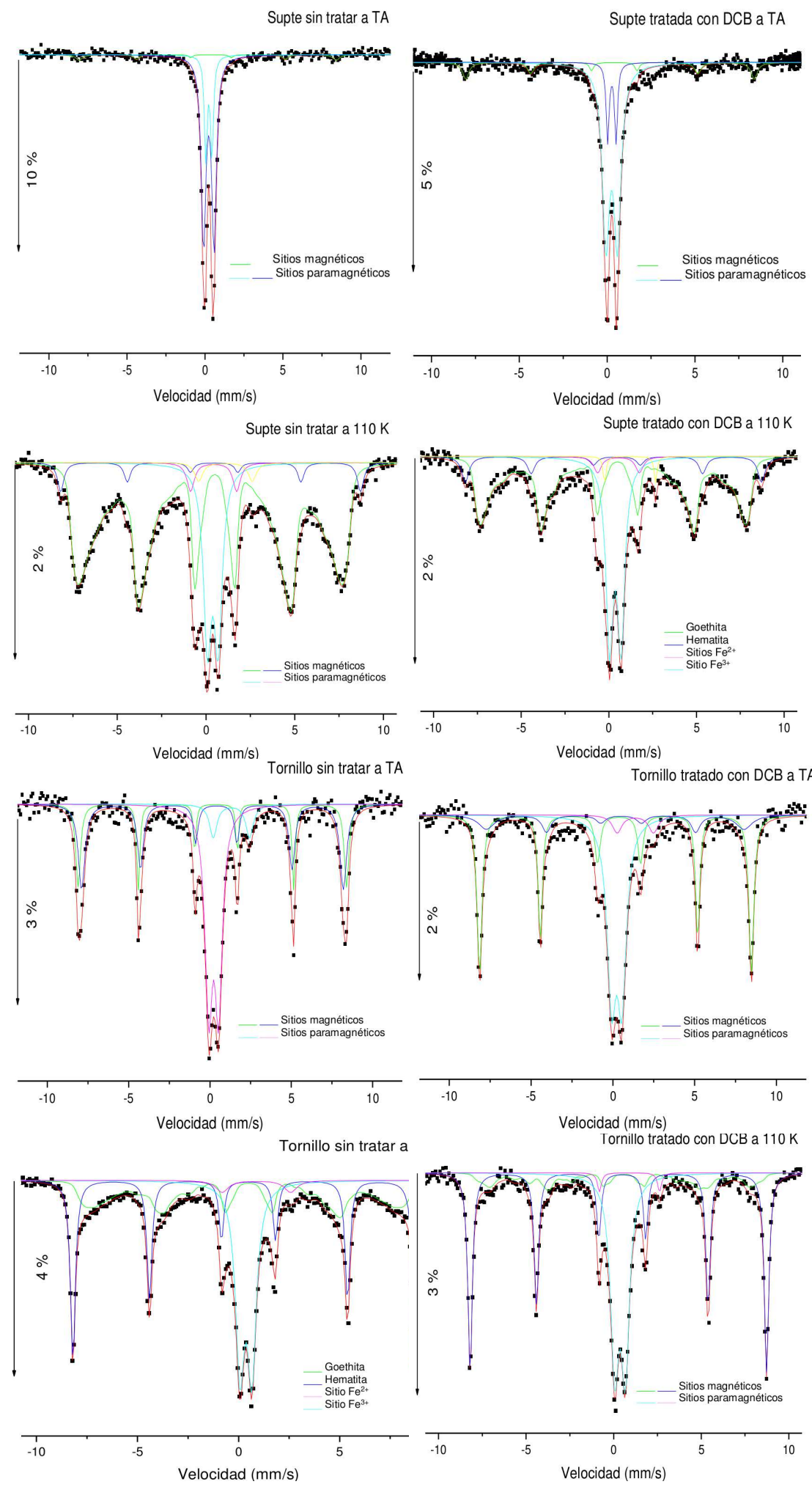

Figura 4: Espectros Mössbauer de la fracción arcilla de las muestras de suelo de Supte y Tornillo. 


\begin{tabular}{|c|c|c|c|c|c|c|}
\hline Asignación & $\delta(\mathrm{mm} / \mathrm{s})$ & $2 \varepsilon(\mathrm{mm} / \mathrm{s})$ & $\Delta \mathrm{E}_{q}(\mathrm{~mm} / \mathrm{s})$ & $\mathrm{B}_{h f}(\mathrm{~T})$ & $\mathrm{A}(\mathrm{mm} / \mathrm{s})$ & $A(\%)$ \\
\hline \multicolumn{7}{|c|}{ Supte sin tratamiento DCB y a $\mathrm{T}=300 \mathrm{~K}$} \\
\hline Fe mag & 0.39 & -0.21 & & 51.10 & 0.010 & 5.69 \\
\hline $\mathrm{Fe}^{3+}$ & 0.37 & & 0.40 & & 0.043 & 24.03 \\
\hline $\mathrm{Fe}^{3+}$ & 0.36 & & 0.67 & & 0.125 & 70.28 \\
\hline \multicolumn{7}{|c|}{ Supte con tratamiento $\mathrm{DCB}$ y a $\mathrm{T}=300 \mathrm{~K}$} \\
\hline Fe mag & 0.40 & -0.25 & & 50.95 & 0.008 & 12.30 \\
\hline $\mathrm{Fe}^{3+}$ & 0.38 & & 0.48 & & 0.009 & 13.59 \\
\hline $\mathrm{Fe}^{3+}$ & 0.37 & & 0.65 & & 0.051 & 74.11 \\
\hline \multicolumn{7}{|c|}{ Supte sin tratamiento DCB y a $\mathrm{T}=110 \mathrm{~K}$} \\
\hline Dist. Gth & 0.48 & -0.25 & & 40.00 & 0.134 & 71.36 \\
\hline Fe mag & 0.47 & -0.20 & & 52.40 & 0.009 & 4.95 \\
\hline $\mathrm{Fe}^{2+}$ & 0.54 & & 2.59 & & 0.005 & 2.89 \\
\hline $\mathrm{Fe}^{2+}$ & 1.19 & & 3.00 & & 0.004 & 2.03 \\
\hline $\mathrm{Fe}^{3+}$ & 0.50 & & 0.60 & & 0.035 & 18.77 \\
\hline \multicolumn{7}{|c|}{ Supte con tratamiento DCB y a $\mathrm{T}=110 \mathrm{~K}$} \\
\hline Dist. Gth & 0.48 & -0.25 & & 40.10 & 0.055 & 55.34 \\
\hline Fe mag & 0.47 & -0.20 & & 52.40 & 0.008 & 8.80 \\
\hline $\mathrm{Fe}^{2+}$ & 0.66 & & 2.40 & & 0.003 & 2.94 \\
\hline $\mathrm{Fe}^{2+}$ & 1.32 & & 3.00 & & 0.002 & 1.80 \\
\hline $\mathrm{Fe}^{3+}$ & 0.48 & & 0.66 & & 0.031 & 31.12 \\
\hline \multicolumn{7}{|c|}{ Tornillo sin tratamiento DCB y a $\mathrm{T}=300 \mathrm{~K}$} \\
\hline Fe mag & 0.37 & -0.23 & & 51.17 & 0.023 & 21.58 \\
\hline Fe mag & 0.38 & -0.23 & & 49.89 & 0.033 & 31.51 \\
\hline $\mathrm{Fe}^{2+}$ & 1.43 & & 2.23 & & 0.007 & 6.91 \\
\hline $\mathrm{Fe}^{3+}$ & 0.34 & & 0.61 & & 0.042 & 40.00 \\
\hline \multicolumn{7}{|c|}{ Tornillo con tratamiento $\mathrm{DCB}$ y a $\mathrm{T}=300 \mathrm{~K}$} \\
\hline Fe mag & 0.38 & -0.21 & & 51.64 & 0.041 & 41.86 \\
\hline Fe mag & 0.42 & -0.34 & & 49.65 & 0.012 & 12.19 \\
\hline $\mathrm{Fe}^{2+}$ & 1.48 & & 2.22 & & 0.004 & 4.61 \\
\hline $\mathrm{Fe}^{3+}$ & 0,35 & & 0,63 & & 0,040 & 41,34 \\
\hline \multicolumn{7}{|c|}{ Tornillo sin tratamiento $\mathrm{DCB}$ y a $\mathrm{T}=110 \mathrm{~K}$} \\
\hline Dist. Gth & 0.50 & -0.23 & & 41.40 & 0.025 & 31.11 \\
\hline Fe mag & 0.49 & -0.22 & & 52.49 & 0.028 & 34.69 \\
\hline $\mathrm{Fe}^{2+}$ & 0.99 & & 3.40 & & 0.002 & 2.93 \\
\hline $\mathrm{Fe}^{3+}$ & 0.48 & & 0.59 & & 0.025 & 31.27 \\
\hline \multicolumn{7}{|c|}{ Tornillo con tratamiento $\mathrm{DCB}$ y a $\mathrm{T}=110 \mathrm{~K}$} \\
\hline Dist. Gth & 0.58 & -0.23 & & 40.40 & 0.017 & 15.72 \\
\hline Fe mag & 0.49 & 0.22 & & 52.49 & 0.048 & 44.21 \\
\hline $\mathrm{Fe}^{2+}$ & 1.00 & & 3.41 & & 0.002 & 1.87 \\
\hline $\mathrm{Fe}^{3+}$ & 0.48 & & 0.59 & & 0.042 & 38.20 \\
\hline
\end{tabular}

Tabla 1: Parámetros hiperfinos Mössbauer a $\mathrm{T}=300 \mathrm{~K}$ y a $\mathrm{T}=110 \mathrm{~K}$ de la fracción arcilla de las muestras de suelos de Supte y Tornillo sin y con tratamiento DCB. Los valores de $\delta$ están referidos al Fe metálico. 


\section{Conclusiones}

En los suelos tropicales como se observa en el caso de la muestra de Tornillo, el efecto de una intensa intemperización causa la pérdida de $\mathrm{Si}$, incrementando la concentración de los sesquióxidos de Fe y Al. Esto podría explicar la diferente composición mineralógica de los dos suelos estudiados.

Los principales sesquióxidos de $\mathrm{Fe}$ encontrados en los suelos estudiados han sido identificados como hematita y goethita. En el caso de la muestra de Supte la hematita aparece como una componente magnética alrededor de cinco veces menor respecto a la muestra de Tornillo.

La efectividad del tratamiento de disolución selectiva sobre los sesquióxidos de Fe es clara, esto puede ser evaluado por la disminución de las áreas de absorción resonante después del tratamiento DCB, particularmente concerniente a la disolución de goethita en el estado superparamagnético para ambas muestras. El doblete intenso de $\mathrm{Fe}^{3+}$, para el caso de la muestra Supte, es asignado a la caolinita y esto es confirmado por los resultados de DRX. En el caso de la muestra de Tornillo, el doblete más intenso podría estar asociado a la moscovita, illita o minerales interestratificados, como se evidencia en DRX.

Los espectros de EMT a TA de las muestras sin tratar indican que la muestra de Supte contiene el doble de hierro que la muestra de Tornillo. Por otro lado, los espectros EMT a $110 \mathrm{~K}$ revelan otra característica contrastante entre las dos muestras por la presencia de una distribución de tamaño de grano más fino para los sesquióxidos de hierro en la muestra de Supte que da lugar a la mayor presencia de efectos superparamagnéticos en esta muestra.

Los espectros tomados a $110 \mathrm{~K}$, para ambas muestras no tratadas y tratadas, revela la existencia de sitios ocupados por $\mathrm{Fe}^{2+}$, los cuales no son visibles en los espectros a TA. Esto podría ser producto de un proceso de transferencia de electrones de valencia (inter valence charge transfer, IVCT) [8]. Esto se puede dar en el caso de un compuesto que contiene cationes vecinos del tipo $\mathrm{A}^{3+} \mathrm{B}^{2+}$ (de valencia mixta) y donde existe la posibilidad de que un electrón de valencia del sitio $B$ sea transferido al sitio $A$, creando $A^{2+} B^{3+}$; ejemplos de compuestos mineralógicos de valencia mixta son: magnetita, ilvaita, y vivianita. Este proceso permite comprender algunas de las propiedades físicas de los minerales de valencia mixta, ya sea que dicho proceso ocurra como manifestación natural o producido químicamente, activo a TA pero suprimido a muy bajas temperaturas [8, 9 ]. Se requiere más estudios sobre este fenómeno.

\section{Agradecimientos}

Los autores agradecen al Departamento de Química de la Universidad Federal de Minas Gerais, Brasil, por la colaboración en la toma de los espectros Mössbauer a 110 $\mathrm{K}$ y la obtención de los difractogramas de rayos $\mathrm{X}$; así también a la Facultad de Ciencias Físicas de la Universidad Nacional Mayor de San Marcos por sus servicios en la toma de los espectros Mössbauer a TA.

\section{Referencias}

[1] M. L. Jackson: Soil chemical analysis, advanced course, second edition, University of Wisconsin, Madison (1985).

[2] D. M. Moore y R. C. Reynolds (Eds.); X-Ray Diffraction and the Identification and Analysis of Clay Materials, Oxford University Press, Oxford (1997).

[3] Dorothy Carroll; Clay Minerals: A guide to their $X$ ray identification, Special paper 126, The Geological Society of America, Boulder (1969).

[4] T. C. Gibbs; Principles of Mössbauer Spectroscopy, Halsted, London (1976).
[5] R. M. Cornell y U. Schwertmann, The iron oxides: Structure, properties, reactions, occurrence and uses, VCH Publishers, New York (1996).

[6] O. P. Mehra y M. L. Jackson; Iron oxide removal from soils and clays by a dithionite-citrate system buffered with sodium bicarbonate; $7^{\text {th }}$ Natl. Conf. on Clays and Clay Minerals, (1960).

[7] R. A. Brand; Normos Mössbauer Fitting Program, User's guide, (1995).

[8] Paul R. Lear y Joseph W. Stucki; Clay and Clay Minerals 35(5), 373 (1987).

[9] R. B. Scorzelli, E. Baggio-Saitovitch y J. Danon; J. Physique 37, C6-801 (1976). 